\title{
Oral health findings, needs and demands of visually impaired children in Saudi Arabia
}

\section{Introduction}

An estimated 253 million people live with vision impairment: 36 million are blind and 217 million have moderate to severe vision impairment (Bourne 2017). Few studies were done in Saudi Arabia on visual impaired population. In 1986 , a revealed that $1.5 \%$ of the Saudi population is fully blind, while $7.8 \%$ is partially blind according to the World Health Organization definition. The etiology of visual impairment varies with different population groups. According to the WHO the most common cause of visual impairment in the developing world is untreated cataract $(43 \%)$ and different ocular disease secondary to diabetes mellitus (24\%). ${ }^{1}$ In developed countries the most common cause of visual impairment is cited as age-related macular degeneration. THe Royal National Institute for the Blind stated that only $8 \%$ of people have a congenital visual impairment with the rest developing a visual impairment through local or systemic disease, accidents or age-related degeneration. ${ }^{2}$ Furthermore, a huge proportion of adults with a visual impairment relate to hearing problems which may require further help to the provision of dental care. ${ }^{3}$ Some of the causes of visual impairment may be associated with other medical problems, such as cardiac defects or diabetes, which are more likely to take an effect on dental care than the visual impairment. ${ }^{4}$ Visual Impairment has an impact on oral health through physical, social, or informational barriers related to impairment, medical condition and associated medical disorders. Visually impaired people are challenged every day in their daily activities. The effects of blindness are many, but one of the most common is the inability of the individual to maintain oral health. ${ }^{5}$ Despite the high prevalence of visually impaired people in Saudi Arabia, there is no sufficient information about oral health in this population. It is essential to assess the oral needs and demands of this under-privilege population for one to be able to set policies and plan for oral health program, the purpose of this study is to assess the oral health status of children with a visual impairment.

\section{Literature Review}

\section{Methods of literature search and appraisal}

The literature search was conducted from October 2017-January 2018 using the Saudi Digital Library. The systematic search was done by carrying out a topic search using the basic search that included related terms "Visual Impairment, Oral Health, Blindness, Caries, Plaque, Orthodontic, Periodontal, Malocclusion, Medical Complication, Dental Surface, and Health Services". The searches were limited to studies published between 1965-2017, English language, and subjects aged $6-15$ years old.

\section{Prevalence of visual impairment in Saudi Arabia}

In Comparison, the prevalence of dental caries and gingivitis are higher among visually impaired children rather that visually impaired adults. ${ }^{6}$ Still other studies have revealed that the oral hygiene of the blind population in general is significantly worse than in an equivalent sighted one. ${ }^{7}$ Another aspect to look at is that patients who have a visual impairment may not pick up on certain non-verbal aspects and may be disadvantaged in regard to this issue. With respect to written
Volume 9 Issue 3 - 2018

\author{
Najla Alghamdi,' Mustafa Alshehri,' Mansour \\ Assery,' Abdulrahman Al Saffan,' Hoda \\ Abdellatif ${ }^{2}$ \\ 'Department of Dentistry, Riyadh Elm University, Saudi Arabia \\ ${ }^{2}$ College of Dentistry, Princess Nourah University, Saudi Arabia
}

Correspondence: Najla Alghamdi, Department of Dentistry, Riyadh Elm University, Riyadh, Saudi Arabia, Tel +966 50140 333I,Email Nmalfhdan@hotmail.com

Received: June 06, 2018 | Published: June 19, 2018

information used in dental services few clinics provide large print/ Braille appointment cards or information sheets. ${ }^{8}$ Of course, some dental treatments might be invasive and perceivably threatening and a visual impairment may make this more so, hence it may be appropriate to commence treatment with short appointments until the patient is accustomed and used to the dental staff and a a rapport is established. ${ }^{9}$

\section{Oral health status}

\section{Caries involvement}

A number of studies have reported Visually Impaired Children (VIC) have a higher caries prevalence than non-Visually Impaired Children (Non-VIC). ${ }^{6,10}$ Consequently, they may be at a higher risk of developing oral diseases which include dental caries or periodontal disease, resulting from a greater difficulty in attaining good oral hygiene. Additionally, it might be due to the visual barrier in gaining the ability to visually assess whether dental plaque has been effectively removed or if their gums bleed while brushing their teeth; dental plaque is an important prerequisite for the development of dental caries and periodontal diseases. ${ }^{11}$ Rationally, visually impaired individuals cannot visualize the plaque on the teeth surface, so even after getting a hold in understanding the importance of oral hygiene it would still be difficult for them which may result in the progression of dental caries as well as inflammatory disease of the periodontium ${ }^{12}$ A study of children suggested that caries load is not affected by visual impairment. ${ }^{13}$ However, there is a positive association between some causes of ocular disease and dental caries, ie. Sjögren's syndrome. ${ }^{14}$

\section{Periodontium \& soft tissues}

Such as caries, periodontal problems are also resulting from poor oral hygiene. In 2003 Nandini et, al made a study which involved a comparison between caries and gingivitis within the visually impaired. It has been shown that thirty-seven percent of the participants were affected by dental caries and seventy-one percent by gingivitis. Visual impairment can have a negative effect upon the individual's oral hygiene, some of them having poorer oral hygiene than sighted peers. As they might have a higher risk in generating periodontal diseases with higher levels of calculus and debris than those who lack a visual impairment. ${ }^{13}$ According to Shetty et al, 2010 sixty-six percent of visually impaired children (VIC) reported difficulty in tooth brushing 
which leads to an inadequate plaque removal and poor oral hygiene. Their visual impairment put them in a disadvantage and more exposed to a higher risk in developing gingival inflammation and dental caries compared to non-VIC. Another issue to discuss is their dental visits, as a study from China found that ninety-two percent of visually impaired patients did not have a regular dentist or attend regularly; consequently, eighty-five percent of those persons have periodontal pockets and thirty-one percent also suffered from deep pockets. ${ }^{11}$ Mucosal lesions may arise in individuals with impaired vision possibly as a consequence of bruxism and lip and cheek biting. ${ }^{15}$ Nevertheless, in an interesting study done by Shah et.al 2004 showed an association highlighted between some congenital ocular defects and gingival fibromatosis/enlargement.

\section{Dental findings}

A study conducted by in 2014 by Bimstein E et al. showed that children and adolescents with or without visual impairment develop similar dental caries prevalence. (Bhat $\mathrm{N}$ et al. 2011) found that visually impaired children (32.5\%) higher prevalence of teeth fracture than that of sighted children (9.6\%). (Varghese RK et al. 2011) found that an overall prevalence of traumatic injuries was $37.8 \%$. Totally blind subjects $(57.1 \%)$ had significantly higher percentage of teeth fracture than that of partially sighted (32\%). Females (43.1\%) were having higher teeth fracture incidences than males $(29.9 \%)$.

\section{Orthodontic needs}

The visually impaired usually exhibit high orthodontic treatment needs because of an increased prevalence and severity of malocclusions. ${ }^{16}$ There is a high motivation to improve the quality of life from the parents to their children; by improving the appearance and the oral function, they are also the least likely to receive orthodontic treatment. ${ }^{17}$ Giving attention to malocclusion in children in general leads to a considerable improvement in the quality of life. ${ }^{18}$ In another study done by Pancham et.al. concluded that a large proportion of the visually impaired children have severe cases of malocclusion which requires mandatory treatment, as they have a higher frequency of malocclusion traits than normal children (Pancham et.al. 2013).

\section{Access to oral health care}

In an estimation of a study group showed that up to $70 \%$ of the participants may be suitable for registration. The Royal National Institute of Blind People (RNIB) ${ }^{2}$ had reported that around two thirds of those who are eligible to register as blind or partially sighted are actually not, which may have affect the suitability in the access to services and additional community and social help. Another issue showed difficulties in accessing dental care including the lack of National Health Service (NHS) general dental practitioners, difficulty locating NHS dental services, and the high expense of dental care. $24 \%$ percent of participants weren't registered with a dentist while $3 \%$ did not know how to obtain dental care services. ${ }^{19}$

\section{Dental treatment}

\section{Preventive measures}

Generally speaking, the oral health of visually impaired people may be overshadowed because of a focus on their disabilities or limited access to oral health care. It has been reported that "dental treatment is the greatest unattended health need of the disabled". Some of the reasons for this may be due to the poor use of dental services and lack of dental awareness, inadequate recall systems, difficulties during practical treatment sessions, and the socio-economic status of the disabled person, pain, underestimation of treatment needs, communication problems and poor patient cooperation. There is a need in improving the access to dental services as well as oral health education is necessary to ensure that best dental health is within the reach of these less fortunate children. On the other hand, removal of plaque is a skill that can be mastered only when the individual has the dexterity to manipulate a toothbrush and an understanding of the objectives of this activity. It is obvious that many visually impaired individuals will find the maintenance of their own oral hygiene much more difficult than normal individuals because those who are blind lack the vision to understand and master the technique of oral hygiene practices. Studies have shown that oral hygiene can be improved significantly by providing an intensified daily brushing by dental personnel, by the development of self-help workshops, by providing effective staff training, or by a combination of all these approaches.

Manish Jain in 2013 suggested that:

1. Primary prevention approaches should be taught to the staff of schools, to the guardians or caregivers and, when appropriate, to the individual children and young adults.

2. Pit and fissure sealants should be applied to the permanent molars and premolars soon after eruption and parents should be advised of the need for regular monitoring and maintenance of fissure sealants.

3. The children should be given suitable toothbrushes, fluoride toothpaste and be shown how to brush their teeth.

4. Fluoride varnish should be applied to any areas of enamel decalcification for children with poor tolerance of dental procedures.

5. Regular school-based programs of toothbrushing should be implemented and reinforced in all these groups with disabilities. Children should be also instructed to clean their teeth twice a day and oral hygiene should be practiced at school and supervised by teachers.

6. Educational institutions should include oral health education as part of their training programs.

7. In-service training in the promotion of good oral health for children with disabilities and in how to access oral care ought to be provided for teachers, institutional staff and parents.

8. Positive links between educational establishments and dental services should be established to promote the oral health of children with disabilities. To enhance oral health outcomes, advanced training is recommended for dental providers and the staff of schools.

A low concentration of fluoride can surprisingly inhibit carbohydrate metabolism of oral streptococci and growth of S. mutans which are associated with the development of dental caries. It could be used as a mouthwash with fluoride concentration which will help in preventing the growth of the MS level among children. ${ }^{20}$ The commonly used fluoride mouthwash is the Sodium Fluoride (NaF) since it's great effectiveness, low cost, ease of formulation and the lack of both; taste and tooth staining (Hind, 1999). Accompanying mechanical movements to mouth rinsing would help achieve a better 
oral hygiene to the individual. As tooth brushing and miswak stick are considered the most reliable and effective mechanical procedures in preventing plaque accumulation if performed on a daily basis. ${ }^{21}$

\section{Parent's responsibilities}

Visually impaired children usually face difficulty in achieving an adequate toothbrushing procedure, this includes the application of the tooth paste on the toothbrush as well as the performance. These strains would be demonstrated by oral hygiene supervision. Furthermore, the lack of knowledge and behavior in oral health by the parents by not getting their children to a regular dental checkup would not help ease the problem of the progression of a poor oral health among the visually impaired children. Manually developing these dexterities for the visually impaired children would allow for a complete control of the process independently without the help of a secondary hand.

\section{Social involvement}

In a recent study ${ }^{22}$ conducted in Saudi Arabia showed that the visually impaired population has a poor plaque control, gingivitis and dental caries, as well as the limitation and difficulties for accessing the health care providers. As they need extensive oral health promotion programs control plaque and prevent caries.

Visual impairment may impact on oral health through physical social or information barrier related to the impairment, attendant medical conditions or lack information in a suitable format. Other difficulties include the lack of transport and services, insufficient resources or financial considerations, lack of social awareness, education and the training of service provider employees. ${ }^{8}$

\section{Purpose of this study}

The purpose of this study is to evaluate the oral health condition within the visually impaired children aged 6-15 in the city of Riyadh, Saudi Arabia.

\section{Specific Objectives:}

1. Assess oral health knowledge among school children in Riyadh

2. Estimate the distribution and severity of major oral diseases and conditions among

3. Estimate the need for care and need for community-oriented disease prevention programs for this selected population

Estimate the nature and urgency of the oral health treatment(s) required in this population.

\section{Methods}

The oral health survey of the visually impaired subjects was held in Riyadh Elm University (REU) - Namuthajiah Campus- in Riyadh. The subjects of this study were within the age of 6-15 years old. It consists of two components, oral health examination and an oral health survey questionnaire. The following paragraphs describe the methods and study design used for each component and the study, overall.

\section{Definition of visual impairment}

According to the 10th revision of the WHO International Statistical Classification of Diseases, Injuries and Causes of Death, 'Low Vision' is defined as visual acuity of less than $6 / 18$ but equal to or better than
$3 / 60$, or a corresponding visual field loss to less than $20^{\circ}$, in the better eye with the best possible correction. 'Blindness' is defined as visual acuity of less than $3 / 60$, or a corresponding visual field loss to less than $10^{\circ}$, in the better eye with the best possible correction. 'Visual impairment' includes both low vision and blindness. Which we targeted in our research.

\section{Study design}

The Survey of the Oral Health Status of Riyadh of visually impaired children employed a multi-stage sample selection. The first stage involved getting a list of all public schools for visually impaired in Riyadh city by the Ministry of Education from which sampling will be carried out. The second stage, letter of introduction was sent to 16 schools in Riyadh. The purpose of the letter was to introduce the study, request permission to conduct the screening and to schedule visits to the clinics of dental school of Riyadh Elm University (Namuthajiah Campus). Once the school directors agreed to participate, arrangement of the school visit date was made. The third stage involved obtaining agreement of all children who are enrolled in the 16 schools to participate in the study in a total of 147 male students and 120 female students.

16-school 120-female 147-male.

\section{Dental examination}

The dental examination was conducted into sites, one at REU dental clinics and at the school sites.

REU dental clinics: The oral health examinations were conducted at the REU dental clinics in two days. Subjects were examined seated in the dental chair, and the examiner assisted by a recorder (dental intern) familiar with the examination coding criteria. The examination was done under LED lighting from the dental chair and used dental examination kits (1 Mouth Mirror, 1 Tweezer, 1 Dental Explorer and 1 WHO Dental Probe). Infection control and waste disposal recommendations and standards were followed. Once the examination is completed, each child received oral hygiene instruction using jaw model. A copy of the screening report was sent to the parents with the child treatment needs results with a list of referral oral health care facilities.

It consisted of two components; both were taken from WHO. It started with hosting the samples in REU for two successive days with the aid of 15 dental interns. They were first taken to a room to be interviewed by dental interns using the WHO Oral Health Questionnaire for Children, after completion they were taken for the dental examination. REU helped by providing two fixed dental chairs (Sirona) to the study site, as well as dental examination kits and jaw models. The examination started by taking samples to the dental chair, a dental intern assisted the examiner by documenting the WHO's oral health assessment form for children. While two samples were examined the others were held with the dental interns demonstrating and educating their oral health by using brushing techniques, flossing and sensing tooth surfaces on jaw models given. The total samples collected in the 2 days were 112 males and 91 females.

\section{Data collection}

Data collection from the subjects participating in the study will be consisted of two parts - an oral health questionnaire, and an oral health examination. The WHO oral health assessment form and oral health questionnaire for children will be used 
Oral health questionnaire: The first part is questionnaire to the student, designed to collect demographic characteristics and oral health knowledge and attitude. The questionnaire will be translated to Arabic and back translated to English by persons well versed with both the languages. The WHO questionnaire will be administered face to face by REU dental interns. A calibration and training for the dental interns was done before the start of the interview to the visually impaired children.

Oral health examination: The second part is the oral health examination, which will include assessments of dentition status, gingival status, enamel fluorosis, dental erosion, dental trauma, oral mucosal lesions, and intervention urgency. Training and calibrating examiner was conducted to ensure uniform interpretation, understanding and application by examiner of the criteria and codes for the various diseases and conditions to be observed and recorded, and that each examiner can examine consistently. Intra- examiner reliability was assessed.

\section{Sample size and study population}

The sample of this study is a convenient sample of students from 16 schools agreed to participate in the study.

\section{Ethical considerations}

In order for a child to participate in the screening, a signed consent was required. The consent form included an introduction to the oral health survey that was sent to the parents/guardians of the schoolchildren. After examination, a report card was given to the child's parent with regard to the child treatment needs assessment.

\section{Data analysis}

\section{Results}

Our sample was summed up in 147 males and 120 female individuals from different primary and middle schools (aged 6-15) in Riyadh, Saudi Arabia. The participants were examined for the presence of any periodontal condition, existence of caries, malocclusions and the presence of fissure sealants. The information was collected through dental examination, majority of the participants were in the age average of 11 years old ((Table 1) (Table 2). Upon examination, most of the participants had degree III of caries according to the Global Oral Health Scale (Figure 2). In the female participants $35 \%$ showed 8 or more teeth with caries (degree III), while males presented $33 \%$ of the same degree. Presence of periodontal condition was relatively different in numbers, as $42 \%$ of the females presented with a periodontal condition, whereas $34 \%$ in the male's counterpart (Figure 3). Presence of fissure sealants was another issue, as it showed $(77 \%$ and $80 \%)$ for both male and female respectively not having any fissure sealants on any teeth surface during their examination. During examination, malocclusion have been noticed in some cases, especially anterior crowding, it was diagnosed in $32 \%$ of the male samples and $22 \%$ in the female samples. After that class II malocclusion by $8.2 \%$ in male samples and $19.2 \%$ in female samples (Figure 1). Almost no cases shown any dental erosions for both males and females. During examination, $96 \%$ males showed no sign of erosion; with a close percentage for females $97 \%$ also didn't show any presence of dental erosion. In general, most diagnosed cases witnessed the need and urgency of intervention. $41 \%$ of male cases needed to be referred for a comprehensive treatment, as well as $23.1 \%$ needed a prompt treatment. While in the female counterpart; it wasn't any further, $42.5 \%$ of female cases needed to be referred to receive a comprehensive treatment while $25 \%$ of the cases needed a prompt treatment.

Table I Need for intervention urgency in the study sample $(\mathrm{N}=267)$

\begin{tabular}{lllll}
\hline $\begin{array}{l}\text { Intervention } \\
\text { urgency }\end{array}$ & Female (120) & $\%$ & Male (147) & $\%$ \\
\hline $\begin{array}{l}\text { No curative treatment } \\
\text { needed }\end{array}$ & 23 & 19.2 & 29 & 19.7 \\
$\begin{array}{l}\text { Preventive or routine } \\
\text { treatment needed }\end{array}$ & 10 & 8.3 & 14 & 9.5 \\
$\begin{array}{l}\text { Prompt treatment } \\
\begin{array}{l}\text { Immediate treatment } \\
\text { due to pain }\end{array}\end{array}$ & 60 & 25 & 34 & 23.1 \\
$\begin{array}{l}\text { Referred for } \\
\text { comprehensive } \\
\text { evaluation }\end{array}$ & 51 & 5 & 10 & 6.8 \\
\hline
\end{tabular}

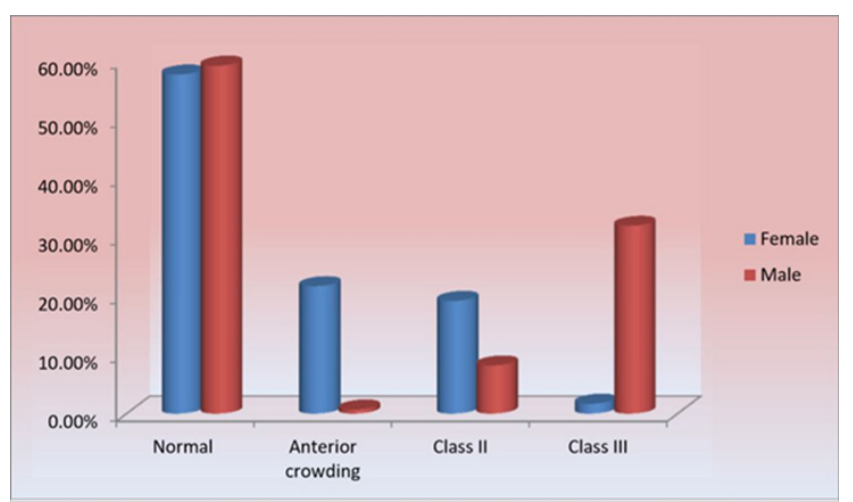

Figure I Presence of mal occlusion in study sample.

Table 2 Socio-demographic characteristics of the study sample $(\mathrm{N}=269)$

\begin{tabular}{lllll}
\hline \multirow{2}{*}{ Variables } & \multicolumn{4}{l}{ The study sample $(\mathbf{n = 2 6 9 )}$} \\
\cline { 2 - 5 } & Female (1 20) & $\%$ & Male (147) & $\%$ \\
\hline Age years: & & & & \\
$06-10$ & 59 & 49.2 & 75 & 51.1 \\
II-15 & 61 & 50.8 & 72 & 48.9 \\
Range & $7-15$ & & $7-14$ & \\
Mean $\pm S D$ & $9.91 \pm 2.41$ & & $9.78 \pm 2.15$ & \\
\hline
\end{tabular}

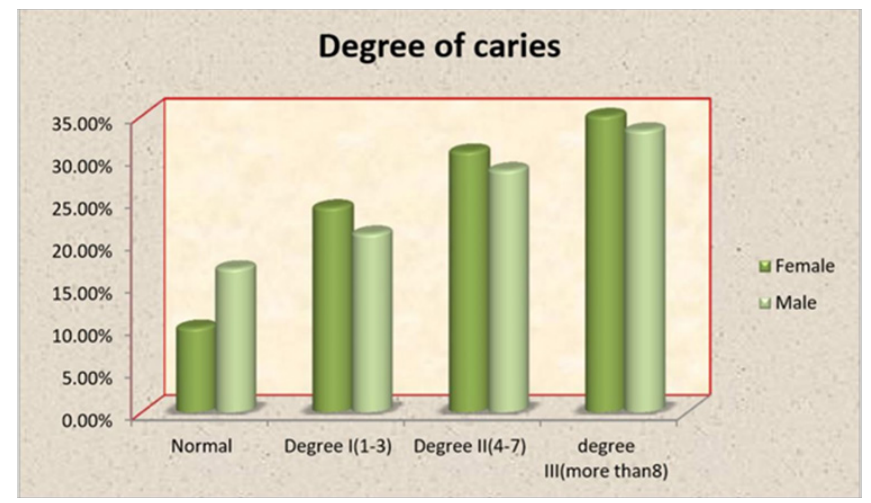

Figure 2 Degree of caries in study sample $(\mathrm{N}=167)$. 


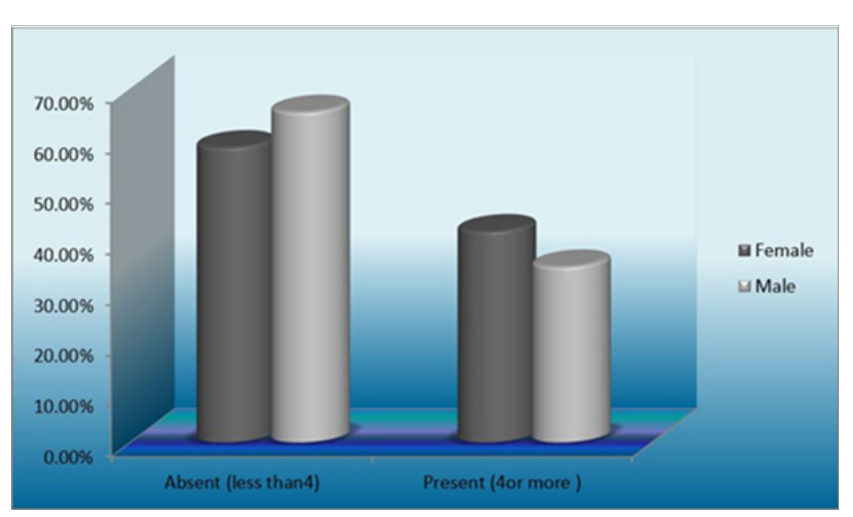

Figure 3 Presence of periodontal conditions in study sample $(\mathrm{N}=120)$.

\section{Discussion}

One of the most important considerations in the human body's health is the oral hygiene health, especially when it comes to the handicapped population. Their limitations could be an obstruction and might interfere with a proper treatment. As prevention is considered the first step in providing a good oral health. In this study, most of the individuals (males and females) showed a high number in different fields of the WHO dental examination, including (Caries, Periodontal conditions and malocclusion) all diagnosed in most of the samples. Starting with degree III of caries according to the Global Oral Health Scale, 33\% and 35\% were shown in both males and females respectively (Figure 2). A similar study done in Indiana University show a higher number of caries experience $48.6 \%$ of their sample had carious teeth (Tagelsir et al. 2013). Periodontal conditions were witnessed in $42 \%$ of the female samples, as well as $34 \%$ of the male samples. Periodontal conditions included the presence of gingival pocketing, swelling, inflamed gums, plaque accumulation or calculus. The numbers documented are considered relatively high comparing the age of the examined samples 9-15 years old (Figure 3). In comparison to another study in 1995, it was shown that $38 \%$ of a Visually impaired sample had bleeding pockets and $48 \%$ of the sample presented with calculus (Bhavasar JP 1995). Most of the examined sample haven't shown any presence of fissure sealants $77 \%$ in males and $80 \%$ in females, not having a preventive barrier at early stage contradicts with what Nawak AJ mentioned in the first paragraph, that prevention is considered the first step in providing a good oral health. Orthodontic consideration has to be considered for the examined samples, their mixed dentition stage showed the complications they're facing by the presence of the anterior crowding and the class II malocclusion cases in both males and females which might interfere with their daily functions and esthetic demands (Figure 1).

\section{Conclusions and recommendation}

Our recommendation is to acknowledge oral health issues of the visually impaired population especially children and adolescents, by providing regular dental checkups to early detect any dental problems including periodontal tissues, dental caries, oral lesions and malocclusions..$^{23-37}$

\section{Acknowledgements}

The Completion of this undertaking could not have been possible without the participation and assistance of so many people, in particular; my parents, my dear husband Dr. Mustafa Alshehri for his love and support and our families for their endless contribution. As well as Prof. Hoda Abdellatif for her measureless aid and supervision, our program director Dr. Navin Ingle for his assistance and REU.

\section{Conflict of interest}

The author declares that there is no conflict of interest.

\section{References}

1. World Health Organization. World Health Report 1998; 2004.

2. Royal National Institute for the Blind; 2004.

3. Lawrence HP, Garcia RI, Essick GK, et al. A longitudinal study of the association between tooth loss and age related hearing loss. Spec Care Dent. 2001;21(4):129-140.

4. EK Mahoney, N Kumar, SR Porter. Effect of visual impairment upon oral health care: a review. Br Dent J. 2008;204(2)63-7.

5. Ishtiaq R, Chaudhary MH, Rana MA, et al. Psychosocial implications of blindness and low vision in students of a school for children with blindness. Pak J Med Sci. 2016;32(2):431-4.

6. Jokic NI, Majstorovic M, Bakarcic D, et al. Dental caries in disabled children. Coll Antropol. 2007;31(1):321-4.

7. Nandini NS. New insights into improving the oral health of visually impaired children. J Indian Soc Pedod Prev Dent. 2003;21(4):142-143.

8. Edwards DM, Merry AJ. Disability part 2: access to dental services for disabled people. A questionnaire survey of dental practices in Merseyside. Br Dent J. 2002;193(5):253-255.

9. Davis RL. The blind dental patient. Ill Dent J. 1965;34:18-21.

10. Al-Qahtani Z, Wyne AH. Caries experience and oral hygiene status of blind, deaf and mentally retarded female children in Riyadh, Saudi Arabia. Odontostomatol Trop. 2004;27(105):37-40.

11. Chang CS, Shih Y. Teaching Oral Hygiene Skills to Elementary Students with Visual Impairments. J Vis Impair Blind. 2005;91(1):26-39.

12. Mann J, Wolnerman JS, Lavie G. Periodontal treatment needs and oral hygiene for institutionalized individuals with handicapping condition. Spec Care Dentist. 1984;4(4):173-176.

13. Greeley CB, Goldstein PA, Forrester DJ. Oral manifestations in a group of blind students. ASDC J Dent Child. 1976;43(1):39-41.

14. Koseki M, Maki Y, Matsukubo T, et al. Salivary flow and its relationship to oral signs and symptoms in patients with dry eyes. Oral Dis. 2004;10(2):75-80.

15. Rapp R, Kanar HL, Nagler B. Pedodontic care for the deaf and blind. Dent Clin North Am. 1966;10:21-34.

16. Winter K, Baccaglini L, Tomar S. A review of malocclusion among individuals with mental and physical disabilities. Spec Care Dentist. 2008;28(1):19-26.

17. Becker A, Chaushu S, Shapira J. Orthodontic treatment for the special needs child. Seminars in orthodontics. 2004;10(4):281-292.

18. Oliveira A, Paiva S, Martins MT. Prevalence and determinant factors of malocclusion in children with special needs. Eur J Orthod. 2011;33(4):413-8.

19. E Watson EK, Moles DR, Kumar N. Online article number E15 Refereed Paper-accepted 19 February 2010. Br Dent J. 2010; 208:E15.

20. Kaneko N, Yoshihara A, Ida H, et al. Influence of a fluoride mouthrinse on mutans streptococci in school children. Caries Res. 2006;40(6):501-7. 
21. Loe H. Oral hygiene in the prevention of caries and periodontal disease. Int Dent J. 2000;50(3):129-139.

22. Aljoharah AA. Oral hygiene practices and periodontal health status of visually impaired Saudi adults in Riyadh, Saudi Arabia. Pak Oral Dent J. 2013;33(1):82-86.

23. Al Sadhan, Asma M Al Jobair, Mariam Bafaqeeh, et al. Dental and medical health status and oral health knowledge among visually impaired and sighted female schoolchildren in Riyadh: a comparative study. BMC Oral Health. 2017;17:154

24. Bhat N, Agrawal A, Nagrajappa R. Teeth fracture among visually impaired and sighted children of 12 and 15 years age groups of Udaipur city, India - a comparative study. Dental Traumatology. 2011;27(5):389-392.

25. Magnitude, temporal trends, and projections of the global prevalence of blindness and distance and near vision impairment: a systematic review and meta-analysis. Lancet Glob Health. 2017;5(9):e888-e897.

26. Freeman R. Communication, body language and dental anxiety. Dent Update. 1992;19(7):307-309.

27. Ismail A Dorout. Knowledge and oral health related behavior among visually impaired subjects in Jazan Region, Kingdom of Saudi Arabia. $J$ Dent and Oral Hygiene. 2015;7(3):33-39.

28. Lebowitz EJ. An introduction to dentistry for the blind. Dent Clin North Am. 1974;18:651-69.

29. Mohan R. Comprehensive Dental Care for the Visually Impaired: a review. International Journal of Oral Health and Medical Research. 2016;3(4):97-101.
30. Ng KKW, Wong MCM, Cheung MMK. Dental care for visually impaired institutionalized older persons in Hong Kong Community Health Project Report Series. Faculty of Dentistry, University of Hong Kong; 2003.

31. O Donnell D, Crosswaite MA. Dental Health Education for the Visually Impaired Child. J Royal Soc Promot Health. 1990;110(2):60-61.

32. Scardina GA, Buonamente A, Messina P. Efficacy of an oral health training programme for visually impaired individuals. Ig Sanita Pubbl. 2008;64:509-16.

33. Schembri A, Fiske J. The implications of visual impairment in an elderly population in recognizing oral disease and maintaining oral health. Spec Care Dent. 2001;21(6):222-226.

34. Scully C, Diz Dios P, Kumar N. Special care in dentistry-handbook of oral healthcare. London: Churchill Livingstone; 2006:1-512.

35. Smutkeeree A, Rojlakkanawong N, Yimcharoen V. A 6-month comparison of toothbrushing efficacy between the horizontal scrub and modified Bass methods in visually impaired students. Int J Paedia Dent. 2011;21(4):278-83.

36. Varghese RK, Agrawal A, Mitra A. Anterior teeth fracture among visually impaired individuals. India Journal of Advanced Dental Research. $2011 ; 2(3): 43-49$

37. World Health Organization. Global Data on Visual Impairments 2010; 2012 . 